\title{
Transliteration and Orthography
}

In developing standards for orthography and transliteration for this volume, we have tried to strike a balance between readability and consistency, while also preserving standard practices in the diverse disciplines from which the research emerges.

For Chinese, Hanyu pinyin is used, with texts transliterated according to other systems standardized according to this system. For Indic languages, the conventions of the International Alphabet for Sanskrit Transliteration have been used, and diacritics retained. Modern place names within the South Asian region, however, have been rendered without diacritics, following conventional English-language spellings (thus Sanchi rather than Sãñ̄ī). Cyrillic names and terms are transcribed according to the widely used modified ALA-LC Romanization system without diacritics (e.g., piatichlenka instead of piatichlenka).

Personal names, toponyms, and terminology from the ancient world are generally rendered in forms that preserve their original orthography as much as possible (e.g., Antiocheia rather than Antioch; Dionysos instead of Dionysus). However, in cases where a word has a generally accepted English spelling, we have followed that convention (Carthage rather than Karthago; Cyrene rather than Kyrene). We have also allowed multiple spellings of certain names and terms, where cross-disciplinary consistency clashed with disciplinary conventions. Thus, we kept Saka alongside Saka and Kuṣanna alongside Kushan. 
